\title{
Cerebrolysin Effects on Cardiac Neuropathy in Diabetic Rats
}

\author{
Enedina Zurita1, Maribel Huerta², Luis De Jesús², Fidel De La Cruz1, Rocio Ortiz-Butron', \\ Gonzalo Flores², Ma De Jesús Gómez-Villalobos²*
}

${ }^{1}$ Depto. de Fisiología, Escuela Nacional de Ciencias Biológicas, IPN, Unidad Profesional Adolfo López Mateos (Zacatenco), Wilfrido Massieu S/N. Col. Nueva Industrial Vallejo, Delegación Gustavo A. Madero. CP 07700., Cd. De México, Mexico ${ }^{2}$ Instituto de Fisiología, 14 Sur, 6301, Col. San Manuel. CP 72570, Benemérita Universidad Autónoma de Puebla, Puebla, Mexico Email: *mgomezvillalobos@aol.com

How to cite this paper: Zurita, E., Huerta, M., De Jesús, L., De La Cruz, F., Ortiz-Butron, R., Flores, G. and De Jesús Gómez-Villalobos, M. (2017) Cerebrolysin Effects on Cardiac Neuropathy in Diabetic Rats. Pharmacology \& Pharmacy, 8, 215-230.

https://doi.org/10.4236/pp.2017.87015

Received: May 25, 2017

Accepted: July 14, 2017

Published: July 17, 2017

Copyright $\odot 2017$ by authors and Scientific Research Publishing Inc. This work is licensed under the Creative Commons Attribution International License (CC BY 4.0).

http://creativecommons.org/licenses/by/4.0/

\begin{abstract}
Autonomic innervation of heart is abnormal in diabetes and produces altered cardiovascular parameters. Cerebrolysin is a neurotrophic factor that improves the dendritic tree and synapses in the central nerve system after brain damage. The aim of this study was to evaluate if cerebrolysin can improve the cardiac neuropathy generated in diabetic rats. Male Sprague-Dawley rats two months old were injected with streptozotocin $(70 \mathrm{mg} / \mathrm{Kg} /$, ip). Hyperglycemia and altered cardiac rate were confirmed after eight weeks of STZ injection, and cerebrolysin treatment was started in control and diabetic rats for two months $(1 \mathrm{ml} / \mathrm{kg} / \mathrm{day}$, ip). Body weight, heart rate, heart rate variability, arterial blood pressure, and blood glucose levels were measured. Also heart weight and levels of nitrites, NGF and VEGF were measured in left ventricle homogenates. The results show that body weight was reduced and blood glucose levels were increased significantly in diabetic rats. Cerebrolysin treatment produced no significant changes in body weight either in blood glucose level in control and diabetic rats. Cerebrolysin treatment in diabetic rats shows an improvement in the altered basal cardiac rate $(306 \pm 6.5 \mathrm{lat} / \mathrm{min})$ compared to diabetic saline group $(272 \pm 8.9$ lat $/ \mathrm{min}: \mathrm{P}<0.05)$, without changes in control rats. Levels of nitrites, VEGF, and NGF in the left ventricle increased in diabetic cerebrolysin treated rats. In conclusion, the results show that cerebrolysin improves some abnormalities observed in the diabetic cardiac neuropathy in rats and suggest that could be considered an additional treatment to prevent or reduce the cardiac autonomic alterations generated in diabetes.
\end{abstract}

\section{Keywords}

Autonomic Innervation, Heart, Hyperglycemia, Cerebrolysin, Rats 


\section{Introduction}

Cardiac neuropathy is an early and common complication of diabetes mellitus and is characterized by abnormalities in the structure and function of sensory and autonomic nerves [1] [2] [3] [4]. Alteration in basal cardiac rate and heart rate variability is often reported in experimental diabetic rats, changes associated with altered diastolic and systolic cardiac functions, and related to abnormal heart innervation that is present after four weeks of induced diabetes for streptozotocin injection in rats [5] [6] [7]. Heart sympathetic and parasympathetic innervations are abnormal in diabetic rats and the magnitude of the deterioration is related to the progression time and severity of hyperglycemia [8] [9]. Histological studies have reported that sympathetic and parasympathetic innervations are altered in the heart after two months of hyperglycemia in rats [10] [11] [12]. Nerve growth factor (NGF) is an essential protein for the development and maintenance of function of the peripheral sympathetic nervous system. A role of NGF in the regulation of sensory and sympathetic neurons has been established and is considered a marker to evaluate sensory innervation [13] [14] [15]. NGF levels are reduced significantly in the hearts of diabetic rats [16] [17]. The administration of NGF in diabetic rats shows evidence of sympathetic reinnervation and regression of cardiac neuropathy that is associated with an improvement in the cardiac functions [18] [19].

Cerebrolysin $(\mathrm{CBL})$ is a neurotrophic factor that has been used in patients as neuroprotection to limit brain damage after strokes [20]. In experimental models of degenerative brain diseases, CBL is able to promote the generation of new functional synapses, improving the integrity of neural circuits in diverse brain structures, which result in the improvement of the cognitive functions, memory, and learning [21] [22] [23]. Recent studies report that cerebrolysin reduces the central and peripheral neuropathy secondary to chronic hyperglycemia induced by streptozotocin in mice and rats [24] [25] [26]. CBL action mechanisms are unclear; however as a compound of many active neuropeptides fragments, it has similar effects of diverse endogenous neurotrophic factors; also a modulation of the pro-NGF/NGF balance was associated with a protective effect on cholinergic neurons in a model of Alzheimer disease in mice [23] [27].

The aim of this study was to determine the beneficial effect of the chronic administration of CBL in rats with established autonomic cardiac neuropathy secondary to diabetes. The effect of cerebrolysin was evaluated in cardiovascular parameters in the integral rats and in the levels of nitrite, nerve growth factor and vascular endothelial growth factor in left ventricle homogenates of control and diabetic rats.

\section{Material and Methods}

Experimental animals were housed in a temperature and humidity controlled environment on a 12-hour light-dark cycles with free access to food and water. All procedures described in this study are in accordance with the "Guide for the Care and Use of Laboratory Animals" of the Mexican Council for Animal Care 
as approved by the BUAP Animal Care Committee. All available measures were taken to minimize animal suffering.

Male Sprague-Dawley rats, weighing $190 \pm 14$ g (two months old), were administered by a single intraperitoneal injection of streptozotocin diluted in saline solution $0.9 \%,(70 \mathrm{mg} / \mathrm{Kg} / \mathrm{ip}, \mathrm{n}=26)$. Other animals matched in age and weight were injected only with the vehicle $(\mathrm{n}=14)$. After 3 days of streptozotocin administration, the glucose level in a fasting state was determined with capillary blood obtained from a minimum section in the distal portion of the tail of the rat using a digital glucometer (Accutrend GC. Roche). The animals were divided into two groups according to glucose blood level; control (glucose concentration, less than $150 \mathrm{mg} / \mathrm{dl}$ ) and diabetic groups (more than $300 \mathrm{mg} / \mathrm{dl}$ ). The groups were followed for two months, after this period, abnormal cardiovascular parameters related to autonomic cardiac neuropathy in rats has been reported. Values of blood glucose in a fasting state were determined again to confirm hyperglycemia, and in accordance with the results animals in both experimental groups were assigned to saline $0.9 \%$ or cerebrolysin treatment. CBL and saline doses were $1 \mathrm{ml} / \mathrm{Kg} /$ day, ip; for two months [23] [25]. Four experimental groups were generated; control plus saline (C-S), control plus cerebrolysin (C-CBL) and diabetic plus saline (D-S) and diabetic plus cerebrolysin (D-CBL) groups. CBL was purchased from EVER Neuro Pharma, GmbH, 4866 Unterach, Austria. Body weight (BW), heart rate (HR), heart rate variability (HRV), systolic and diastolic arterial blood pressure (SABP, DABP), and blood glucose levels in fasting state were determined and recorded in basal conditions (before STZ or saline injection), at two months of hyperglycemia evolution (after STZ or saline injection), and after four months of hyperglycemia evolution and two months of CBL or saline treatment. At the end of the protocol, the rats were deeply anesthetized with sodium pentobarbital ( $40 \mathrm{mg} / \mathrm{Kg}$, ip) and the hearts were quickly removed and washed with Krebs solution. Hearts were quickly frozen in cooled alcohol methane and then stored at $-80^{\circ} \mathrm{C}$, until use.

\subsection{Cardiovascular Parameters}

Systolic and diastolic arterial blood pressure and heart rate and heart rate variability were determined in conscious animals through the tail-cuff plethysmography method with the XBP-1000 Kent Scientific system. Briefly, the rat was collocated in an acrylic restrainer box, in which the tail is outside. Occlusion cuff and sensor volume pressure (plethysmography) were positioned in the tail. The signals were acquired and processed with a limited AD-100 PC oscilloscope, PICO Technology. A minimum of six cycles of inflation and deflation for each determination were made. Systolic arterial blood pressure (SABP) corresponds to the beginning of the arterial pulse recording, after the release of occlusion pressure, and diastolic arterial blood (DABP) to the nadir of the volume curve [28]. Systolic and diastolic arterial blood pressure are reported in $\mathrm{mm} \mathrm{Hg}$. Arterial pulse intervals (P-P intervals) were measured in msec in 30 consecutive arterial pulses in each rat separately, and after mean, standard deviation and 
standard error were determined with Grad Pad software system. The heart rate was obtained through a mean P-P interval in each rat, the mean interval P-P was divided between 1000 and then multiplied by 60 . The mean heart rate was determined in each experimental group and reported in beats/min. The standard deviation values from 30 consecutive P-P intervals in each rat and then averaged in each experimental group was considered as a great estimation of the heart rate variability.

\subsection{Tissue Homogenates}

Samples were taken from frozen hearts, weighing $100 \mathrm{mg}$ from the left ventricle, and processed for protein quantitation, biochemical and immunoassay studies. Briefly, tissues were homogenized in cold phosphate buffer (three sessions of 10 seconds at $30,000 \mathrm{rpm}$ ) and centrifuged at $3000 \mathrm{rpm}$ for 5 minutes at $4^{\circ} \mathrm{C}$. The supernatant was centrifuged at $20,000 \mathrm{rpm}$ for 20 minutes at $4^{\circ} \mathrm{C}$. The pellet was resuspended in cold phosphate buffer and centrifuged again at 20,000 rpm for 20 minutes at $4^{\circ} \mathrm{C}$. Pellets were used for protein quantification and biochemical and immunoassay studies. Protein content was made through the BCA assay protocol from Pierce. The standard curve was made with bovine serum albumin.

\subsection{Nitrite Levels}

Nitrite levels were measured in homogenates from the left ventricle to assess the nitric oxide (NO) production in hearts. The reaction was made with $50 \mu \mathrm{l}$ aliquots of the sample and $50 \mu \mathrm{l}$ of Griess reagent (from SIGMA-ALDRICH, Cat number G4410), in a 96-well plate. Samples were incubated at $22^{\circ} \mathrm{C}$ for $10 \mathrm{mi}$ nutes. Absorbance was measured at $570 \mathrm{~nm}$ in a Synergy HT-Bioteck spectrophotometer. The standard curve was made with sodium nitrite $\left(\mathrm{NaNO}_{2}\right)$.

\subsection{Immunoassays Studies}

The ELISA (enzyme-linked immunosorbent assay) was made for the quantitative measurements of diverse proteins in left ventricle homogenate samples from the different experimental animal groups. Neural growth factor (NGF) and vascular endothelial growth factor (VEGF) were measured using commercial ELISA Kits and processed according to manufacturer instructions. Beta-NGF-Elisa Kit Rab0381-lot 70213 and Rat VEGF-Elisa kit Rab0512-lot number 0423B0713 were from SIGMA-ALDRICH ${ }^{\mathrm{TM}}$. Briefly, we used a specific capture antibody coated on the 96-well plate. Standards and samples were placed in duplicate into the wells, covered and incubated overnight at $4^{\circ} \mathrm{C}$. Afterward, the solution was discarded and the wells were washed with a wash buffer four times. Samples were incubated with biotinylated detection antibody specific, for two $h$ at $22^{\circ} \mathrm{C}$ with shaking. Wells have washed again with a wash buffer four times. After washing away unbound biotinylated antibody, HRP-conjugated streptavidin was applied to the wells, during one $\mathrm{h}$ at $22^{\circ} \mathrm{C}$ with shaking. The washing periods were done again and then, a TMB substrate solution was added to the wells and incubated for 30 minutes at $22^{\circ} \mathrm{C}$, with shaking in a dark room. Excess of the 
volume was discarded and the stop solution was added, changing the color from blue to yellow, and the intensity of the color was measured immediately at 450 $\mathrm{nm}$ for NGF and VEGF. The optical density was measured, in a Synergy HTBioteck microplate reader. Calculation of mean absorbance was made for each set of duplicate standards and samples subtracting the average 0 of standard optical density. The standard curve was plotted with Graph-Pad Prism 6 software, and concentration in the samples was estimated with a linear regression.

\section{Data Analysis}

The data are expressed in absolute values as a mean \pm standard error. Differences between experimental groups were determined using a one-way analysis of variance (ANOVA), followed by the Newman-Keuls Multiple Comparison Test, with Graph Pad Prism 6. P $<0.05$ was considered significantly different.

\subsection{Results}

\subsubsection{Body Weight and Blood Glucose Measurements}

Basal values in the body weight (g) and blood glucose levels in $\mathrm{mg} / \mathrm{dl}$, at the beginning of the protocol and before streptozotocin injection were similar in the control and diabetic rats (Table 1). After two months of streptozotocin injection and before the saline and cerebrolysin treatment, body weight was significantly reduced in the diabetic rats compared to the control rats (body weight in g: 394 \pm 5 , and $251 \pm 10.61$ in control and diabetic rats respectively, $\mathrm{P}<0.05$ ) (Table 1 ). The reduced body weight in the diabetic rats persisted after two months of cerebrolysin and saline treatment, compared to treated control rats (body weight in g: $460 \pm 14.82$, and $471 \pm 9.21$ in C-S and C-CBL rats and $268 \pm 11.79$, and $254 \pm$

Table 1. Different parameters in control and diabetic rats, in basal conditions at two months old before and after streptozotocin administration where the rats were four months old. Changes observed in the parameters are secondary to the hyperglycemia induced by the streptozotocin administration.

\begin{tabular}{|c|c|c|c|c|}
\hline Parameter & $\begin{array}{c}\text { C-rats } \mathrm{n}=14 \\
\text { basal conditions }\end{array}$ & $\begin{array}{c}\text { D-rats } \mathrm{n}=26 \\
\text { basal conditions } \\
\text { before } 2 \text { months } \\
\text { of STZ }\end{array}$ & $\begin{array}{c}\text { C-rats } \mathrm{n}=14 \\
\text { after two months } \\
\text { of vehicle }\end{array}$ & $\begin{array}{c}\text { D-rats } n=26 \\
\text { after } 2 \text { months of } \\
\text { STZ }\end{array}$ \\
\hline Body weight (g) & $209 \pm 4.26$ & $205 \pm 2.8$ & $394 \pm 5$ & $251 \pm 10.61^{*}$ \\
\hline Blood glucose (mg/dl) & $116 \pm 4.92$ & $122 \pm 5.58$ & $138 \pm 7.53$ & $498 \pm 29.78^{*}$ \\
\hline P-P interval (msec) & $129 \pm 4.74$ & $128 \pm 2.5$ & $151 \pm 0.86$ & $191 \pm 0.75^{\star}$ \\
\hline $\begin{array}{l}\text { P-P interval (msec) } \\
\text { standard desviation }\end{array}$ & $11 \pm 1.27$ & $11 \pm 2.5$ & $16 \pm 0.55$ & $10 \pm 2.48^{*}$ \\
\hline Heart rate (beat/min) & $463 \pm 12.8$ & $471 \pm 9.23$ & $387 \pm 11.6$ & $307 \pm 8.16^{*}$ \\
\hline $\mathrm{SABP}(\mathrm{mmHg})$ & $137 \pm 8.42$ & $140 \pm 8.91$ & $146 \pm 8.42$ & $164 \pm 4.94^{*}$ \\
\hline $\mathrm{DABP}(\mathrm{mmHg})$ & $83 \pm 4.2$ & $86 \pm 5.18$ & $89 \pm 6.2$ & $114 \pm 4.47^{\star}$ \\
\hline
\end{tabular}

Data are expressed as mean $\pm \mathrm{SE} .{ }^{\star} \mathrm{P}<0.05$ between control and diabetic rats. C: control. D: diabetic. SD: standard deviation. SABP: systolic arterial blood pressure. DABP: diastolic arterial blood pressure. 
10.12 in $\mathrm{D}-\mathrm{S}$ and $\mathrm{D}-\mathrm{CBL}$ rats respectively, $\mathrm{P}<0.05)$. No significant changes in the body weight were observed according to the saline or cerebrolysin treatment in the control and diabetic groups (Table 2).

Blood glucose levels significantly increased in diabetic rats compared to the control rats after two months of streptozotocin injection and before the vehicle and CBL treatment (blood glucose levels in $\mathrm{mg} / \mathrm{dl}: 138 \pm 7.53$, and $498 \pm 29.78$ in control and diabetic rats respectively, $\mathrm{P}<0.05$ ) (Table 1 ). After vehicle and CBL treatment during two months, and before sacrifice of the experimental animals, blood glucose levels were normal and similar in the control-saline and control-cerebrolysin treated groups (blood glucose levels in $\mathrm{mg} / \mathrm{dl}: 151 \pm 5.86$, and $156 \pm 5.71$ in C-S and C-CBL rats respectively, NS (Table 2), whereas in the diabetic-saline and diabetic-cerebrolysin treated groups, significant increases in blood glucose levels were found (blood glucose levels in $\mathrm{mg} / \mathrm{dl}: 466 \pm 31.21$, and $461 \pm 26.19$ in D-S and D-CBL rats respectively, NS (Table 2). No difference in blood glucose levels was found, in accordance with the vehicle or CBL treatment in the experimental groups.

\subsubsection{Cardiovascular Parameters}

Basal values of cardiovascular parameters at the beginning of the protocol and before streptozotocin injection were similar in the experimental groups (Table 1). After two months of streptozotocin injection and before the vehicle or CBL treatment, heart rate showed a significant decrease in diabetic rats compared to

Table 2. Different parameters in control and diabetic rats showing the effect of cerebrolysin treatment. Rats were 6 months old, with four months of hyperglycemia, and CBL or saline treatment in the last two months. Results show significant differences between the control and diabetic groups in all the parameters evaluated, except for the standard deviation of the P-P interval. However, in accordance with the treatment saline or CBL in the diabetic group, there was a significant difference in the heart rate and in the duration of the P-P interval between saline and CBL treated diabetic rats.

\begin{tabular}{|c|c|c|c|c|}
\hline Parameter & $\begin{array}{l}\text { C-S rats } n=8 \\
\text { after } 2 \text { months of } \\
\text { vehicle }\end{array}$ & $\begin{array}{l}\text { C-CBL rats } n=6 \\
\text { after } 2 \text { months of } \\
\text { CBL }\end{array}$ & $\begin{array}{l}\text { D-S rats } n=11 \\
\text { after } 2 \text { months of } \\
\text { vehicle }\end{array}$ & $\begin{array}{c}\text { D-CBL rats } \mathrm{n}= \\
15 \text { after } 2 \text { months } \\
\text { of } \mathrm{CBL}\end{array}$ \\
\hline Body weight (g) & $460 \pm 14.82$ & $471 \pm 9.21$ & $268 \pm 11.79^{*}$ & $254 \pm 10.12^{\star}$ \\
\hline Heart weight (g) & $1.45 \pm 0.11$ & $1.5 \pm 0.10$ & $1.18 \pm 0.07^{*}$ & $1.16 \pm 0.057^{*}$ \\
\hline Blood glucose mg/dl & $151 \pm 5.86$ & $156 \pm 5.71$ & $466 \pm 31.21^{*}$ & $461 \pm 26.19^{*}$ \\
\hline $\begin{array}{c}\text { Relation heart } \\
\text { wt/body wt (\%) }\end{array}$ & 0.315 & 0.318 & $0.430^{*}$ & $0.450^{*}$ \\
\hline P-P interval (msec) & $161 \pm 0.95$ & $164 \pm 1.43$ & $220 \pm 0.68^{*}$ & $192 \pm 0.82^{*^{\bullet}}$ \\
\hline $\begin{array}{l}\text { P-P interval (msec) } \\
\text { standard deviation }\end{array}$ & $18 \pm 0.93$ & $14 \pm 1.06$ & $14 \pm 0.71$ & $15 \pm 0.68$ \\
\hline Hear rate $($ beat/min) & $362 \pm 12.95$ & $367 \pm 8.44$ & $272 \pm 7.99^{*}$ & $306 \pm 6.51^{*^{\bullet}}$ \\
\hline $\mathrm{SABP}(\mathrm{mmHg})$ & $138 \pm 3.14$ & $140 \pm 2.88^{\star}$ & $156 \pm 7.09^{*}$ & $162 \pm 6.91^{\star}$ \\
\hline DABP (mmHg). & $88 \pm 2.5$ & $93 \pm 3.33$ & $104 \pm 2.74^{*}$ & $96 \pm 3.38^{*}$ \\
\hline
\end{tabular}

Data are expressed as mean $\pm \mathrm{SE}$. ${ }^{\star} \mathrm{P}<0.05$ between control and diabetic rats, in the independent form of the treatment. ${ }^{\circ} \mathrm{P}<0.05$ between saline or cerebrolysin treated rats. C: control. D: diabetic. S: saline. CBL: cerebrolysin. SABP: systolic arterial blood pressure. DABP: diastolic arterial blood pressure. 
the control rats (heart rate in beat/min: $387 \pm 11.6$ and $307 \pm 8.16$; control against diabetic rats, $\mathrm{P}<0.05)$. According to the changes observed in the basal heart rate, the P-P interval duration also showed significant changes in the diabetic rats compared to the control rats (P-P interval in msec: $151 \pm 0.86$ against $191 \pm 0.75$; control against diabetic rats, $\mathrm{P}<0.05)($ Table 1 , Figure $1(\mathrm{a}))$. These values were preserved and accentuated after two months of saline treatment in the diabetic rats, however in the diabetic-CBL treated rats, there was a significant increase in the heart rate compared to the diabetic-saline treated rats (heart rate in beat/min: $272 \pm 7.99$ in $\mathrm{D}-\mathrm{S}$ and $306 \pm 6.51$ in $\mathrm{D}-\mathrm{CBL}$ rats; $\mathrm{P}<0.05$ ) (Figure 1(b), Table 2). Also, the changes were observed in the P-P interval duration in the diabetic-saline rats compared to the diabetic-cerebrolysin treated rats (P-P interval in msec: $220 \pm 0.68$ in $\mathrm{D}-\mathrm{S}$ and $192 \pm 0.82$ in $\mathrm{D}-\mathrm{CBL}$ rats; $\mathrm{P}<0.05$ ) (Table 2, Figure 1(b)). Heart rate variability evaluated throughout standard deviation of the average of P-P interval in each experimental group show significant changes only in the diabetic rats compared to the control group before the saline or $\mathrm{CBL}$ treatment (HRV in msec: $16 \pm 0.55$ against $10 \pm 2.48$; control against diabetic rats, $\mathrm{P}<0.05$ ) (Table 1 ). In this study, CBL or saline treatment during two months did not generate significant changes in the heart rate variability in the experimental groups (Table 2). Systolic and diastolic arterial blood pressure showed a significant increase in the diabetic rats, compared to the control rats only after two months of streptozotocin injection (SABP in mmHg: 146 \pm 8.42 in control group and $164 \pm 4.94$ in diabetic group; $\mathrm{P}<0.05)$. DABP in mmHg: $89 \pm 6.2$ in control group and $114 \pm 4.47$ in diabetic group; $\mathrm{P}<0.05$ (Table 1). These differences were sustained after two months of saline or cerebrolysin treatment, showing no significant differences between saline and cerebrolysin treated groups (Table 2).

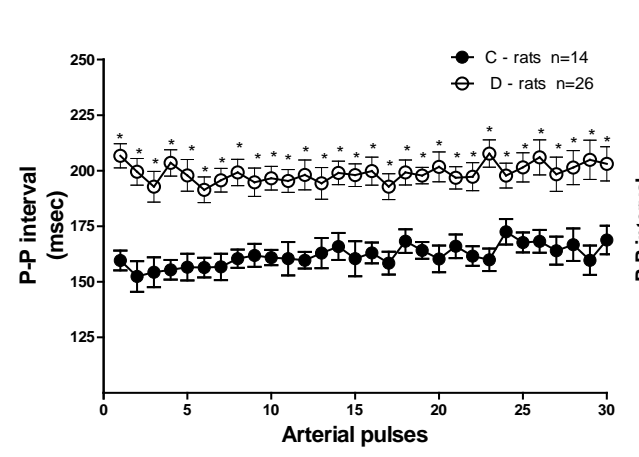

(a)

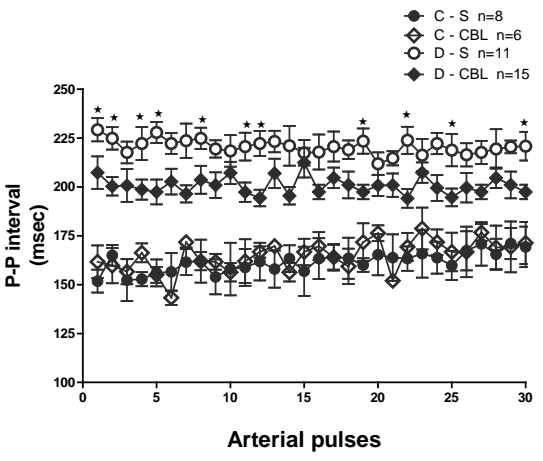

(b)

Figure 1. Arterial pulse interval and their variability in control and diabetic rats before and after the saline or CBL treatment. (a) shows the changes in the duration of interval $\mathrm{P}$-P expressed as mean $\pm \mathrm{SE}$, in rats 4 months old with two months of hyperglycemia and without any treatment; (b) shows the changes in the duration of interval P-P expressed as mean $\pm \mathrm{SE}$, in 6-month-old rats with four months of hyperglycemia and with saline or CBL treatment during the last two months. Arterial pulse interval duration was significantly increased in the diabetic rats compared to the control rats. It was clear that $\mathrm{CBL}$ treatment decreased these changes in the hyperglycemic rats. ${ }^{*} \mathrm{P}<0.05$ between control and diabetic rats, in the independent form of the treatment. 


\subsubsection{Heart Weight and Ratio Body Weight/Heart Weight}

Wet heart weight was reduced in the diabetic rats compared to control rats (wet heart weight in g: $1.45 \pm 0.11$, and $1.5 \pm 0.10$ in C-S and C-CBL rats and $1.18 \pm$ 0.07 , and $1.16 \pm 0.05$ in D-S and D-CBL rats respectively, $\mathrm{P}<0.05$ between control and diabetic rats). However, the heart weigh/body weight ratio was higher in the diabetic rats compared to control rats (heart weight/body weight ratio in \%: $0.315 \pm 0.011$, and $0.318 \pm 0.010$ in C-S and C-CBL rats and $0.430 \pm 0.07$, and $0.450 \pm 0.05$ in D-S or D-CBL rats respectively, $\mathrm{P}<0.05$ between control and diabetic rats). No significant changes in the wet weight heart either in the heart weigh/body weight/ratio were observed according to the saline or cerebrolysin treatment in the experimental groups (Table 2).

\subsubsection{Nitrite Levels}

Nitrite levels in the left ventricle homogenates from the diabetic saline treated rats were increased compared to the control saline group (C-S: $8.56 \pm 0.24$ and $\mathrm{D}-\mathrm{S}: 15.95 \pm 1.58$, in $\mu \mathrm{M} / \mathrm{L} ; \mathrm{P}<0.05)$. However, nitrite levels also increased in the control and diabetic CBL treated rats (C-CBL: $17.4 \pm 2.27$ and D-CBL: 19.13 \pm 2.56 ), without significant changes between them (Figure 2(a)).

\subsection{Immunoassay Results}

\subsubsection{Neural Growth Factor Levels}

Levels of NGF were reduced in diabetic saline treated rats compared to the control saline treated rats (C-S: $3.58 \pm 0.142$ versus $D-S: 2.30 \pm 0.106$ in $\mathrm{ng} / \mathrm{g} \mathrm{w}$. weight, $\mathrm{P}<0.05)$. However, the NGF levels increased significantly in the diabetic CBL treated rats compared to the levels in the diabetic saline treated rats, (D-S: $2.30 \pm 0.106$ versus D-CBL: $4.102 \pm 0.187 \mathrm{ng} / \mathrm{g} \mathrm{w}$-weight, $\mathrm{P}<0.05)$. Also in the control, CBL treated rats, there was an increase in the NGF levels compared to the control saline rats (C-S: $3.58 \pm 0.142$ against C-CBL: $4.33 \pm 0.395 \mathrm{ng} / \mathrm{g} \mathrm{w}$. weight, NS) (Figure 2(b)).

\subsubsection{Vascular Endothelial Growth Factor Levels}

VEGF levels in left the ventricle homogenates were reduced in diabetic saline treated rats compared to the control saline treated rats (C-S: $0.159 \pm 0.013$ versus $\mathrm{D}-\mathrm{S}: 0.113 \pm 0.010$, in $\mathrm{ng} / \mathrm{g}$ w.weight; $\mathrm{P}<0.05)$. However, VEGF levels significantly increased in the diabetic CBL treated rats compared to the diabetic saline treated rats (D-S: $0.113 \pm 0.010$, versus D-CBL: $0.207 \pm 0.007 \mathrm{ng} / \mathrm{g}$ w.weight, $\mathrm{P}<$ $0.05)$. Also in the control-CBL treated group there was a small but not a significant increase in the levels of the VEGF (C-S: $0.159 \pm 0.013$ against C-CBL: 0.182 $\pm 0.006 \mathrm{ng} / \mathrm{g} \mathrm{w} \cdot$ weight. NS) (Figure 2(c)).

\section{Discussion}

The results show that cerebrolysin administration in rats with findings of established cardiac neuropathy reduces the alterations in cardiovascular parameters and some biochemical markers generated after four months of hyperglycemia induced by streptozotocin injection in rats, suggesting that cerebrolysin has 


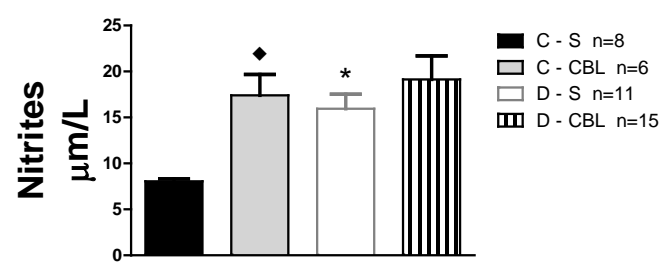

(a)

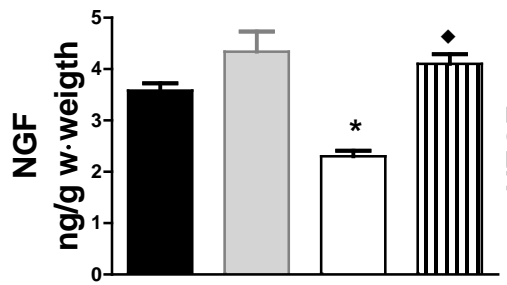

(b)

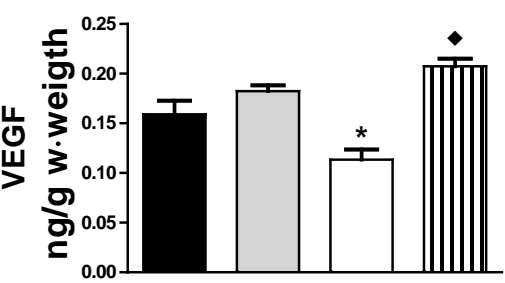

(c)

Figure 2. Graphs show the results related to the effect of CBL treatment on the levels of nitrites, NGF and VEGF, in left ventricle homogenates in control and diabetic rats. (a) Effect of the CBL on nitrites levels in left ventricle homogenates in the experimental groups. Nitrite levels were increased significantly in diabetic-saline rats compared to control-saline rats. However, an increase in nitrite levels was also observed in the control and diabetic groups treated with the cerebrolysin; (b). CBL treatment on NGF levels in left ventricle homogenates in the experimental groups; (c). BL treatment on VEGF levels in left ventricle homogenates in the experimental groups. The CBL treatment in diabetic rats increased significantly the levels of NGF and VEGF compared to diabetic rats plus vehicle. Four rats in each group. ${ }^{\star} \mathrm{P}<0.05$ between control and diabetic rats, in the independent form of the treatment. ${ }^{\star} \mathrm{P}<0.05$ between saline or cerebrolysin treated rats.

beneficial effects on cardiac neuropathy associated with diabetes.

Chronic hyperglycemia induced by streptozotocin produces a significant decrease in the body weight of the experimental animals [5] [6] [7]. In our study, we also found a significant decrease in the body weight of diabetic rats compared to control rats. The changes in the body weight were confirmed at two and four months of hyperglycemia and no changes were generated with the saline or cerebrolysin treatment in the experimental groups. Heart weight at the end of the protocol also was significantly different between the control and diabetic rats; however, there was no change in according to the saline or cerebrolysin treatment in the experimental groups. These results suggest that in diabetic rats, four months after streptozotocin injection, CBL administration at a dose of $1 \mathrm{ml} / \mathrm{kg}$ does not induce a significant change in the body weight and heart weight. Blood glucose levels were high as expected after streptozotocin injection and sustained until the end of the protocol. The results show no significant changes in the blood glucose levels with the administration of saline or cerebrolysin in the control and diabetic treated groups. These results demonstrate that cerebrolysin does not generate changes in blood glucose levels neither control nor diabetic rats. However, it has been reported that CBL is able to increase the expression of glucose transporter (Glut1) in the blood-brain barrier in rats and then could improve the use of glucose for the cells [29]. 
Diabetic neuropathy is a frequent and early complication of diabetes, and evolution time of diabetes and a lack of glycemic control are major risk factors for neuropathy [8] [9]. More than half of all individuals with diabetes eventually develop neuropathy, and neuropathy increases mortality [1] [2] [3] [4]. Neuropathy in diabetes affects selectively sensory and autonomic neurons. The neuropathy of the heart in diabetes is associated with a reduction in axon number, swollen mitochondria, accumulation of neurofilaments and microtubules, and glycogen and vacuoles [3]. Longer autonomic nerves, such as vagus that determine $75 \%$ of parasympathetic activity, could be affected early in diabetes. Vagal impairment seen in early diabetes leads to a relative predominance of sympathetic activity in sympatho-vagal balance [8] [12]. In the early stages of chronic hyperglycemia, an increase in release and in the heart content of noradrenaline is reported, with the opposite changes in the late stages [9] [10] [11]. Then it is currently accepted that cardiac autonomic neuropathy is associated with parasympathetic withdrawal, sympathetic dominance in early stages and a decreased sympathetic and parasympathetic tone in late stages.

Basal heart rate is a functional parameter that depends on the balance between sympathetic and parasympathetic discharge. Several studies have reported that after two months of hyperglycemia there is structural and functional evidence of cardiac neuropathy in streptozotocin rats, such as a reduced basal cardiac rate, altered cardiac rate variability, and decreased stores of catecholamines in the heart, some alterations that we confirmed in our study [5]-[12]. It is very well documented that a resting heart rate in diabetic rats is decreased compared to control rats and a loss of autonomic balance is the main mechanism involved [5] [6] [7]. In the present study, the basal heart rates, and heart rate variability were significantly reduced in the diabetic group compared to the control group, before saline or cerebrolysin treatment. In addition, systolic and diastolic arterial blood pressure was significantly higher in the same group compared to the control group. The changes observed in arterial blood pressure and heart rate variability in diabetic rats are compatible with an increased sympathetic tone, whereas the reduced basal heart rate suggests a predominant parasympathetic tone influenced on heart rate. In conclusion at this stage, the experimental group injected with streptozotocin developed cardiac neuropathy. Once cardiac neuropathy was established, the rats were treated with cerebrolysin.

The altered basal heart rate was persistent after four months of hyperglycemia in diabetic rats with the saline administration, whereas in the diabetic-CBL treated rats an improvement in the basal cardiac rate was observed. Basal heart rate in diabetic-CBL treated rats was significantly higher compared to the diabetic-saline treated rats (heart rate in beat/min: $272 \pm 7.99$ against $306 \pm 6.51 ; \mathrm{P}$ $<0.05$, diabetic-saline against the diabetic-CBL group). Cerebrolysin treatment during two months did not generate significant changes in the heart rate variability, in neither systolic nor diastolic arterial blood pressure in the experimental groups. Failure to find differences in heart rate variability at this stage may have been because the HRV was determined only with 30 consecutive arterial pulses 
and it has been reported that a minimum must be for 5 minutes in rats. The results suggest that CBL could partially reduce the autonomic imbalance produced in cardiac diabetic neuropathy, probably through an improvement in heart sympathetic innervation. Additional studies focused on sympathetic innervations are necessary to confirm these results.

Recently, changes in the heart autonomic innervation have been related to changes in the expression and levels of neurotrophic factors. Nerve growth factor (NGF) is a protein that promotes survival and differentiation of nerve cells not only in the central nervous system but also in the autonomic and the sensory nervous system [13] [16] [19]. NGF belongs to the neurotrophin family and their effects on the cells are through the activation of TrkA receptors. TrkA is a receptor tyrosine kinase and p75, a low-affinity neurotrophin receptor, which mediates the NGF effects on the cells. NGF is expressed in the heart, particularly on the surface of sympathetic neurons [16]. NGF regulates growth and synapses and differentiation in sympathetic neurons and enhances neuritic growth and target innervations. It also has acute effects, modulating the sympathetic synapses in the heart and producing a long-term enhancement of synaptic transmission in cultures of sympathetic neurons and cardiac myocytes [16]. Levels of NGF in the heart are considered a marker of the integrity of sympathetic nerve cells into the heart [16] [19]. NGF levels in a normal heart have been reported from $0.34 \pm 0.02$ to $10 \pm 0.6 \mathrm{ng} / \mathrm{g}$ w.weight. Differences in values are related to the time of hyperglycemia evolution and the cardiac chamber studied [14] [15] [16] [17]. Abnormal NGF, expression, levels, and transport have been reported in experimental models of diabetes in mice and rats [1] [18]. Levels of NGF have been reported to be decreased in diabetic cardiac neuropathy and the alterations of cardiac sensory innervation in the diabetic heart are explained by decreases in NGF [17]. Furthermore, restitution of NGF levels through gene transfer of NGF or administration of synthetic NGF in mouse and rats with cardiac diabetic neuropathy was associated with an improvement in the impaired sensory innervation and improved the function of diabetic hearts [18] [19]. The results suggest the ability of NGF to promote regeneration of noradrenergic nerves that is lost in diabetes. In association with the results described, in the present study, we found similar findings related to the levels of NGF in the left ventricle from the hearts of diabetic rats. In the left ventricle homogenates from rats with four months of hyperglycemia evolution, the NGF levels were reduced compared to the NGF levels in the control group. Interestingly the administration of CBL during two months increased NGF levels significantly in the left ventricle homogenates from the diabetic rats, and then we could suggest that sympathetic innervation is also improving in the heart of hyperglycemic rats since an improvement in the cardiac rate was observed.

Vascular endothelial dysfunction is an early and common alteration in diabetes and one mechanism involved is the abnormal stimulated and basal production of nitric oxide (NO) [30] [31]. One of the most important functions of vascular endothelium is related to the production of $\mathrm{NO}$ to regulate the vascular 
tone. NO is generated in endothelial cells by activation of the nitric oxide synthase (NOS) and diffuses into the smooth muscle to activate the soluble guanylyl cyclase (sGC), producing an increase in the intracellular cyclic guanonosine-3', 5'-monophosphate concentration, leading to relaxation [30] [31] [32]. There are three NOS isoforms, endothelial (eNOS), inducible (iNOS), and neuronal (nNOS), which are involved in the production of NO [30] [31] [32]. Cardiac levels of nitrite, an indirect parameter of the NO concentration, have been reported to be normal, increased or decreased in hearts from diabetic rats. The different results are related to the evolution time and severity of hyperglycemia. In rats with 4 - 8 weeks of hyperglycemia, increased nitrite levels have been reported [32] [33]. However, decreased levels are found in the long time evolution of diabetes [32]. Alteration in the expression of the isoforms of nitric oxide synthases (NOS) has been also reported in the hearts of diabetic rats. Inducible nitric oxide synthase (iNOS) was found increased and associated with an increase in the nitrite levels in left ventricle homogenates from the hearts of diabetic rats [32] [33] [34] [35]. However, a decrease in the content of nNOS and a decrease in the nNOS immunostaining were found in sections of peripheral nerve bundles from diabetic rats [31]. In the present study nitrite levels in left ventricle homogenates from diabetic rats were increased in the hyperglycemic saline and CBL treated rats. An increase in nitrite level in the hyperglycemic saline treated rats could be explained by an upregulation of iNOS reported previously in diabetic rats [33] [34]. Mechanisms that explain the increase of nitrite levels with CBL administration could be explained through the direct effect on iNOS of cerebrolysin. Further studies must be done to confirm the present results.

Micro and macro vascular angiopathy is a constant pathological finding in humans and animals with diabetes [30] [34]. The microangiopathy also impairs nerve perfusion and could contribute to neuropathy or nerve degeneration. VEGF is a main signal derived from endothelial vascular cells that stimulate the formation of new blood vessels [36] [37]. Downregulation of VEGF expression preceded the onset of microvascular endothelial cell apoptosis and a subsequent cascade of events that produce systolic and diastolic heart failure in diabetic hearts [36] [37]. Levels of VEGF, VEGF mRNA levels and expression of VEGF receptors (VEGF-R1 (Flt-1) and VEGF R-2 (KDR) are reduced in the hearts of diabetic rats [36] [37]. Plasma VEGF levels also were reduced in diabetic rats $(71.3 \pm 13 \mathrm{pg} / \mathrm{ml})$, compared to nondiabetic rats $(86 \pm 13 \mathrm{pg} / \mathrm{ml})$ [37]. In addition, restitution of VEGF in diabetic rats through gene therapy, administration of VEGF, or endothelin antagonist drugs, produces an increase in the capillary density, reduces cardiomyocyte apoptosis, prevents the impairment of sensory nerves by restoration of neural vascularity and the cardiac function is improved [38] [39]. In this study, we found a close correlation with the levels of NGF, similar changes in the levels of VEGF. VEGF levels were reduced significantly in the heart from diabetic rats with saline treatment, however in the cerebrolysin treated diabetic rats there was a recovery in the VEGF levels.

Cerebrolysin is a complex mix of peptides. It has been reported that CBL contains 630 peptides, the main component fragments being of tubulin-alpha and 
beta chain, actin, and myelin basic protein [21] [23]. Although it appears that CBL does not produce changes in glucose blood levels in normoglycemic and hyperglycemic conditions and in consequences, it does not improve insulin production; there is some evidence that suggests that CBL could improve the utility of glucose for the cells. Previous studies report that CBL improves the expression of Glut 1 in cells and this could be a mechanism through which CBL appears to improve glucose metabolism [29]. In one study where CBL was administered after four weeks of hyperglycemia-induced by streptozotocin to rats in doses of $2.5 \mathrm{ml} / \mathrm{Kg} /$ day, CBL reduced the levels of serum TNF- $\alpha$, and nitrites in diabetic rats and preserved the neuronal cells in the hippocampus [24]. A reduced level of nitrites in the brain is explained by an inhibition of the iNOS [24]. The previous reports show that CBL is able to partially reverse the nerve cell damage secondary to hyperglycemia, particularly in the hippocampus [24]. CBL improves the survival of grafted neural stem cells into the hippocampus, in a murine model of Alzheimer disease. The mechanisms proposed are by a reduced number of caspase- 3 and tunnel positive cells, with an increase of BDNF [23]. Recent studies in experimental models of diabetes in mice and rats also report an improvement in function and structures of central and peripheral nerve system with the cerebrolysin administration [25] [26]. The neuroprotective effects of CBL may involve multiple mechanisms, including signal regulation and expression of proteins, neurotrophic factors; a reduction of oxidative stress, inflammation, abnormal neurotransmission, and apoptosis [23] [24] [39] [40]. Action mechanisms proposed are related mainly to the peptides that simulate the action of neural growth factors [21] [23] [27]. Recent reports demonstrate that CBL increases the levels of neurotrophins as BDNF (Brain-derived neurotrophic factor) and NGF in several regions from the brain [23] [27]. In the present study, administration of CBL was able to increase the levels of NGF and VEGF in the hearts from diabetic rats, values that were reduced in heart of diabetic rats with the saline administration. The cerebrolysin mechanisms involved could be related to their capacity to work as neurotrophic factor generating a cascade of events that could improve heart innervation. Further studies must be done to confirm the mechanisms involved in the described effects of cerebrolysin in the diabetic cardiac neuropathy.

\section{Conclusion}

In conclusion, cerebrolysin administration improved the basal heart rate and restored the levels of NGF and VEGF that were reduced in the left ventricle homogenates from rats with established diabetic cardiac neuropathy. Taken together, the results suggest that cerebrolysin is able to reduce some abnormalities observed in the cardiac autonomic neuropathy secondary to diabetes, and then could be considered as an additional therapeutic approach in diabetes mellitus, focused on patients with cardiac autonomic neuropathy.

\section{Acknowledgements}

The financial support for this study was with VIEP-BUAP grants (GOVM- 
SAL12-14). We are grateful to Professor Robert Simpson for editing this English-language text.

\section{References}

[1] Vinik, A.I. and Ziegler, D. (2007) Diabetic Cardiovascular Autonomic Neuropathy. Circulation, 115, 387-397. https://doi.org/10.1161/CIRCULATIONAHA.106.634949

[2] Kuehl, M. (2012) Cardiovascular Autonomic Neuropathies as Complications of Diabetes Mellitus. Nature Reviews Endocrinology, 8, 405-416.

https://doi.org/10.1038/nrendo.2012.21

[3] Sanyal, S.N., Wada, T., Yamabe, M., Anai, H., Miyamota, S., Shimada, T. and Ono, K. (2012) Synaptic Degradation of Cardiac Autonomic Nerves in StreptozotocinInduced Diabetic Rats. Pathophysiology, 19, 209-307. https://doi.org/10.1016/j.pathophys.2012.08.002

[4] Forbes, J.M. and Cooper, M.E. (2013) Mechanisms of Diabetic Complications. Physiological Reviews, 93, 137-188. https://doi.org/10.1152/physrev.00045.2011

[5] Giudice, P.L., Careddu, A., Magni, G., et al. (2002) Autonomic Neuropathy in Streptozotocin Diabetic Rats: Effect of Acetyl-L-Carnitine. Diabetes Research and Clinical Practice, 56, 173-180. https://doi.org/10.1016/S0168-8227(01)00375-8

[6] Li, X., Jiang, Y.-H., Jiang, P., et al. (2015) Analisis of Heart Rate Variability and Cardiac Autonomic Nerve Remodeling in Streptozotocin-Induced Diabetic Rats. Experimental and Clinical Endocrinology and Diabetes, 123, 272-281. https://doi.org/10.1055/s-0035-1547258

[7] Hicks, K.K., Seifen, E., Stimers, J. and Kennedy, R.H. (1998) Effects of Streptozotocin-Induced Diabetes on Heart Rate, Blood Pressure, and Cardiac Autonomic Nervous System. Autonomic Neuroscience, 121, 7-15.

[8] Lin, Y.D., Hsu, K.L., Wu, E.T., et al. (2008) Autonomic Neuropathy Precedes Cardiovascular Dysfunction in Rats with Diabetes. European journal of Clinical Investigation, 38, 607-614. https://doi.org/10.1111/j.1365-2362.2008.01992.x

[9] Xuan, Y.-L., Wang, Y., Xue, M., et al. (2015). In Rats, the Duration of Diabetes Influences Its Impact on Cardiac Autonomic Innervations and Electrophysiology. Autonomic Neuroscience: Basic and Clinical, 189, 31-36. https://doi.org/10.1016/j.autneu.2015.01.003

[10] Felten, S.Y., Peterson, R.G., Shea, P.A., Besch Jr., H.R. and Felten, D.L. (1982) Effects of Streptozotocin Diabetes on the Noradrenergic Innervation of the Rat Heart: A Longitudinal Histofluorescence and Neurochemical Study. Brain Research $\mathrm{Bu}$ lletin, 8, 593-607. https://doi.org/10.1016/0361-9230(82)90086-7

[11] Kuncová, J., Sviglerová, J., Tonar, Z. and Slavikova, J. (2005) Heterogeneous Changes in Neuropeptide Y, Norepinephrine and Epinephrine Concentrations in the Heart of Diabetic Rats.

[12] Lund, D.D., Subieta, A.R., Pardini, B.J. and Chang, K.S. (1992) Alterations in Cardiac Parasympathetic Índices in STZ-Induced Diabetic Rats. Diabetes, 41, 160-166. https://doi.org/10.2337/diab.41.2.160

[13] Govoni, S., Pascale, A., Amadio, M., et al. (2011) NGF and Heart: Is There a Role in Heart Disease? Pharmacological Research, 63, 266-277.

[14] Korsching, S. and Thoenen, H. (1983) Nerve Growth Factor in Sympathetic Ganglia and Corresponding Target Organs of the Rat: Correlation with Density of Sympathetic Innervation. Proceedings of the National Academy of Sciences, 80, 35133516. https://doi.org/10.1073/pnas.80.11.3513 
[15] Xing, J., Lu, J. and Li, J. (2014) Nerve Growth Factor Decreases in Sympathetic and Sensory Nerves of Rats with Chronic Heart Failure. Neurochemical Research, 39, 1564-1570. https://doi.org/10.1007/s11064-014-1348-5

[16] Lokhart, S.T., Turrigiano, G.G. and Birren, S.J. (1997) Nerve Growth Factor Modulates Synaptic Transmission between Sympathetic Neurons and Cardiac Myocytes. Journal of Neuroscience, 17, 9573-9582.

[17] Schmid, H., Forman, L.A., Cao, X., Sherman, P.S. and Stevens, M.J. (1999) Heterogeneous Cardiac Sympathetic Denervation and Decreased Myocardial Nerve Growth Factor in Streptozotocin-Induced Diabetic Rats. Implications for Cardiac Sympathetic Dysinnervation Complicating Diabetes. Diabetes, 48, 603-608. https://doi.org/10.2337/diabetes.48.3.603

[18] Vo, P.A. and Tomlinson, D.R. (1999) The Regeneration of Peripheral Noradrenergic Nerves after Chemical Sympathectomy in Diabetic Rats: Effects of Nerve Growth Factor. Experimental Neurology, 157, 127-134. https://doi.org/10.1006/exnr.1999.7018

[19] Ieda, M., Kanazawa, H., Ieda, Y., Kimura, K., Matsumura, K., et al. (2006) Nerve Growth Factor Is Critical for Cardiac Sensory Innervation and Rescues Neuropathy in Diabetic Hearts. Circulation, 114, 2351-2363. https://doi.org/10.1161/CIRCULATIONAHA.106.627588

[20] Heiss, W.D., Brainin, M., Bornstein, N.M., et al. (2012) Cerebrolysin in Patients with Acute Ischemic Stroke in Asia. Results of a Doublé-Blind, Placebo-Controlled Randomized Trial. Stroke, 43, 630-636. https://doi.org/10.1161/STROKEAHA.111.628537

[21] Rockenstein, E., Mante, M., Adame, A., Crews, L., Moessler, H. and Masliah, E. (2007) Effects of Cerebrolysin TM on Neurogenesis in an APP Transgenic Model of Alzheimer's Disease. Acta Neuropathologica, 113, 265-275. https://doi.org/10.1007/s00401-006-0166-5

[22] Solis-Gaspar, C., Vazquez-Roque, R., Gómez-Villalobos, M.J. and Flores, G. (2016) Cerebrolysin Improves Memory and Ameliorates the Neural Atrophy of Spontaneous Hypertension Aged Rat. Synapse, 70, 378-389. https://doi.org/10.1002/syn.21912

[23] Rockenstein, E., Desplats, P., Ubhi, K., et al. (2015) Neuropeptide Treatment with Cerebrolysin Improves the Survival of Neural Stem Cells Grafts in an APP Transgenic Model of Alzheimer Disease. Stem Cell Research, 15, 56-67.

[24] Georgy, G.S., Nassar, N.N., Mansour, H.A. and Abdallah, D.M. (2013) Cerebrolysin Ameliorates Cognitive Deficits in Type III Diabetic Rats. PLoS ONE, e64647.

[25] Sanchez-Vega, L., Juarez, I., Gómez Villalobos, M.J. and Flores, G. (2015) Cerebrolysin Reverses Hippocampal Neural Atrophy in Mice Models of Diabetes Mellitus Type 1. Synapse, 69, 326-335. https://doi.org/10.1002/syn.21819

[26] Dong, H.Y., Jiang, X.M., Niu, C.B., Du, L., Feng, J.Y. and Jia, F.Y. (2016) Cerebrolysin Improves Sciatic Nerve Dysfunction in a Mouse Model of Diabetic Peripheral Neuropathy. Neural Regeneration Research, 11, 156-162.

https://doi.org/10.4103/1673-5374.175063

[27] Ubhi, K., Rockenstein, E., Vazquez Roque, R., Mante, M., et al. (2013) Cerebrolysin Modulates Nerve Growth Factor/Nerve Growth Factor Ratio and Ameliorates the Cholinergic Deficit in a Transgenic Model of Alzheimer's Disease. Journal of Neuroscience Research, 91, 167-177. https://doi.org/10.1002/jnr.23142

[28] Feng, M., Whitesall, S., Zhang, Y., Beibel, M., D’Alecy, L. and DiPetrillo, K. (2008) Validation of Volume-Pressure Recording Tail-Cuff Blood Pressure Measurements. American Journal of Hypertension, 12, 1268-1291. 
https://doi.org/10.1038/ajh.2008.301

[29] Gschanes, A., Boado, R., Sametz, W. and Windisch, M. (2000) The Drug Cerebrolysin and Its Peptide Fraction E021 Increases the Abundance of the Blood-Brain Barrier GLUT1 Glucose Transporter in Brain of Young and Old Rats. The Histochemical Journal, 32, 71-77. https://doi.org/10.1023/A:1004003008683

[30] Pieper, G.M. (1998) Review of Alterations in Endothelial Nitric Oxide Production in Diabetes. Protective Role of Arginine on Endothelial Dysfunction. Hypertension, 31, 1047-1060. https://doi.org/10.1161/01.HYP.31.5.1047

[31] Cellek, S., Foxwell, N. and Moncada, S. (2003) Two Phases of Nitrergic Neuropathy in Streptozotocin-Induced Diabetic Rats. Diabetes, 52, 2353-2362. https://doi.org/10.2337/diabetes.52.9.2353

[32] Stockklauser-Farber, K., Ballhausen, T. and Rosen, P. (2000) Influences of Diabetes on Cardiac Nitric Oxide Synthase Expression and Activity. Biochimica et Biophysica Acta, 1535, 10-20.

[33] Soliman, H., Craig, G.P., Nagareddy, P., et al. (2008) Role of Inducible Nitric Oxide Synthase in Induction of RhoA Expression in Hearts from Diabetic Rats. Cardiovascular Research, 79, 322-330. https://doi.org/10.1093/cvr/cvn095

[34] Nagareddy, P.R., Xia, Z., McNeill, J.H. and MacLeod, K.M. (2005) Increased Expression of iNOS Is Associated with Endothelial Dysfunction and Impaired Pressure Responsiveness in Streptozotocin-Induced Diabetes. American Journal of Physiology. Heart and Circulatory Physiology, 289, H2144-H2152. https://doi.org/10.1152/ajpheart.00591.2005

[35] Khanna, S., Singh, G.U. and Khullar, M. (2014) Nitric Oxide Synthase and Diabetic Cardiomyopathy. Nitric Oxide, 43, 29-34.

[36] Han, B., Baliga, R., Huang, H., Giannone, P.J. and Bauer, J.A. (2009) Decreased Cardiac Expression of Vascular Endothelial Growth Factor and Redox Imbalance in Murine Diabetic Cardiomyopathy. American Journal of Physiology. Heart and Circulatory Physiology, 297, H829-H835. https://doi.org/10.1152/ajpheart.00222.2009

[37] Chou, E., Suzuma, I., Way, J.K., et al. (2002) Decreased Cardiac Expression of Vascular Endothelial Growth Factor and Its Receptors in Insulin-Resistant and Diabetic States. A Possible Explanation for Impaired Collateral Formation in Cardiac Tissue. Circulation, 105, 373-379. https://doi.org/10.1161/hc0302.102143

[38] Yoon, Y., Uchida, S., Masuo, O., et al. (2005) Progressive Attenuation of Myocardial Vascular Endothelial Growth Factor Expression Is a Seminal Event in Diabetic Cardiomyopathy. Restoration of Microvascular Homeostasis and Recovery of Cardiac Function in Diabetic Cardiomyopathy after Replenishment of Local Vascular Endothelial Growth Factor. Circulation, 111, 2073-2085. https://doi.org/10.1161/01.CIR.0000162472.52990.36

[39] Jesmin, S., Zaedi, S., Shimojo, N., et al. (2007) Endothelin Antagonism Normalizes VEGF Signaling and Cardiac Function in STZ-Induced Diabetic Rat Hearts. American Journal of Physiology_Endocrinology and Metabolism, 292, E1030-E1040. https://doi.org/10.1152/ajpendo.00517.2006

[40] Patockova, J., Krsiak, M., Marhol, P. and Tumova, E. (2003) Cerebrolysin Inhibits Lipid Peroxidation Induced by Insulin Hypoglycemia in the Brain and Heart of Mice. Physiological Research, 52, 455-460. 
Submit or recommend next manuscript to SCIRP and we will provide best service for you:

Accepting pre-submission inquiries through Email, Facebook, LinkedIn, Twitter, etc. A wide selection of journals (inclusive of 9 subjects, more than 200 journals)

Providing 24-hour high-quality service

User-friendly online submission system

Fair and swift peer-review system

Efficient typesetting and proofreading procedure

Display of the result of downloads and visits, as well as the number of cited articles Maximum dissemination of your research work

Submit your manuscript at: http://papersubmission.scirp.org/

Or contact pp@scirp.org 